\title{
Estudo comparativo de ambientes gamificados no auxílio à aprendizagem
}

\author{
Isolda B. Pereira ${ }^{1}$, Jucelio S. dos Santos ${ }^{2}$, Pablo R. Suarez ${ }^{1}$, Milena N. Alves de Sousa ${ }^{3}$ \\ ${ }^{1}$ Universidade Estadual da Paraíba (UEPB) \\ ${ }^{2}$ Universidade Federal de Campina Grande (UFCG) \\ ${ }^{3}$ Faculdades Integradas de Patos (FIP) \\ isoldapereira2@gmail.com, juceliodcopin.ufcg.edu.br \\ prsuarez@gmail.com, minualsa@hotmail.com
}

\begin{abstract}
The objective of this paper is to perform a survey and comparison of gamma environments based on leaderboards that could be used as a motivational practice capable of impacting the resolution of exercises and, therefore, the learning process of the students. The method adopted in this investigation is an exploratory Survey, in which a questionnaire was applied aiming at an analysis by the researcher of the following environments: Kahoot, GoConqr, Busuu and Duolingo. As results, it was possible to perceive that, although there are some gamificado platforms that allow a learning to develop activities of any discipline, currently there are no tools that respond to all the requirements raised in an ideal way.
\end{abstract}

Resumo. $O$ objetivo deste trabalho é realizar um levantamento e uma comparação de ambientes gamificados baseados em leaderboards que pudessem ser utilizados como uma prática motivacional capaz de impactar na resolução de exercícios e, por conseguinte, no processo de aprendizagem dos discentes. O método adotado nesta investigação é um Survey exploratório, em que foi aplicado um questionário visando uma análise, por parte do pesquisador, dos seguintes ambientes gamificado: Kahoot, GoConqr, Busuu e Duolingo. Como resultados, foi possivel perceber que, apesar de existirem algumas plataformas gamificadas que possibilitem uma aprendizagem para o desenvolvimento de atividades de qualquer disciplina, atualmente não há ferramentas que respondam a todos os requisitos levantados de maneira ideal.

\section{Introdução}

Nos cursos de graduação, todas as disciplinas são de extrema importância para a formação do aluno, desde as mais básicas até as mais complexas. Porém, o que não se pode ignorar são as dificuldades enfrentadas pelos acadêmicos nas disciplinas de difícil compreensão, na assimilação de conteúdos e na resolução de problemas, causando, portanto, baixo rendimento e até mesmo evasão nos cursos. Em uma pesquisa realizada pelos cursos de graduação do Centro de Ciências Exatas e de Tecnologia da Universidade Estadual de Maringá (UEM), foi observado que tanto o problema de reprovação, bem como os critérios de avaliação nas disciplinas de difícil compreensão podem levar os alunos a desistirem do curso [Fregoneis 2002]. 
VIII Congresso Brasileiro de Informática na Educação (CBIE 2019)

Anais do XXV Workshop de Informática na Escola (WIE 2019)

Este insucesso não está apenas relacionado a 'nota baixa' ou a reprovação, mas sim, o fracasso em não entender a importância dos componentes curriculares para os cursos. Sendo assim, lecionar alguma matéria é um desafio para os professores, pois os alunos imaturamente a vem com receio de que são disciplinas de difícil compreensão. Como aborda [Camboim 2017] percebe-se que no Ensino Superior, diversas disciplinas apresentam altos índices de reprovação. Outro fato que pode fazer com que esse problema aumente esses índices é que, se o acadêmico não tiver uma dedicação para com os conteúdos abordados em disciplinas mais complexas que são fundamentais no início de algum curso e que servem como base para todo o percurso da graduação, o seu desempenho pode ser comprometido, pois uma disciplina pode ter relação com outras.

Segundo [Oliveira et al. 2017] é relevante explicar algum tipo de assunto ou currículo no sistema educacional de um país, porém não é um trabalho simples. As dificuldades dependem de cada aluno, quando estão aprendendo um assunto que não tem relação diretamente para a vida. Qualquer educador do mundo que já tenha experiência com o magistério, já pode ter ouvido a pergunta: "Professor (a) porque eu tenho que estudar isso?" Quando se trata de teorias: "Qual a finalidade desse estudo para minha vida?" Tais indagações, infelizmente não são com o intuito de serem respondidas para tirar alguma dúvida, independentemente de qual área for, contudo, o interlocutor está sempre habilitado para replicar a explicação. Essas atitudes facilitam significativamente o 'desastre' da aprendizagem em uma sala de aula.

Diante dessa situação, levanta-se um prognóstico, sobre os reais motivos desses problemas: Os alunos dedicam-se com mais afinco às disciplinas específicas do curso, gerando um grande problema, pois por consequência de não se dedicarem na proporção que as disciplinas exigem, acabam adquirindo um prejuízo na formação acadêmica. Os universitários têm uma concepção de que existem matérias 'específicas' e 'não específicas', ainda que isso não seja uma realidade oficial, o que gera uma falta de interesse dos alunos para as disciplinas que eles chamam 'não específicas'.

Perante este cenário, parte-se do pressuposto que usar estratégias lúdicas como os jogos possa motivar e encorajar comportamentos específicos tais como: mudanças nos comportamentos dos indivíduos para que os mesmos possam desenvolver suas atividades de uma maneira confortável diante das situações que se encontram para dar apoio ao processo do aprendizado acadêmico, minimizando o baixo rendimento, reprovação e até mesmo a evasão.

Diversas técnicas podem incentivar os estudantes no processo de aprendizagem, dentre elas, uma que pode ser abordada é a competição que faz com que o usuário se sinta motivado a participar de uma disputa, faz parte da categoria de estratégias (procedimento da gamificação que está relacionado com alguma ação realizada pelo o usuário). Os pontos, as medalhas e os rankings, chamados Points, Badges and Leaderboards (PBL) são os elementos de gratificações que a gamificação usa, e foram os que mais deixaram resultados positivos, pois, essa metodologia procura motivar/instigar os usuários mostrando-lhes sua posição por meio de uma tabela classificatória [Aires and Leite 2017].

Assim, de acordo com o cenário apresentado, a problemática associada a esse trabalho contempla a seguinte questão: "dentre os ambientes gamificados disponíveis que dão suporte às abordagens apresentadas aos discentes para a resolução de exercícios, qual 
VIII Congresso Brasileiro de Informática na Educação (CBIE 2019)

Anais do XXV Workshop de Informática na Escola (WIE 2019)

o que, por meio do uso dos leaderboards, apresenta melhor potencial capaz de motivar o processo de aprendizagem dos estudantes?".

Portanto, para responder à pergunta anteriormente elencada, esta pesquisa se propôs à realização de um levantamento e de uma comparação de ambientes gamificados baseados em leaderboards que pudessem ser utilizados como uma prática motivacional capaz de impactar na resolução dos exercícios e, por conseguinte, no processo de aprendizagem dos discentes.

Para alcançar tal intuito foi realizado inicialmente o levantamento, por meio de um estudo bibliográfico, de alguns conceitos relacionados ao entendimento da pesquisa, dentre os quais a gamificação, seus desafios e limitações, ambientes gamificados com foco nos leaderboards e nos conceitos relacionados, como competições e rankings, de modo que o cenário e suas potencialidades e restrições pudessem ser melhor compreendidos. Em seguida, foram elencados os ambientes gamificados considerados no estudo, bem como definidos critérios para que a comparação propriamente dita pudesse, enfim, ser realizada.

\section{Método}

A natureza desta pesquisa é aplicada, pois envolve interesses locais, tendo como objetivo gerar conhecimentos para aplicação prática. Em relação ao objetivo, esta pesquisa é classificada como descritiva, visando descrever características ou estabelecer relação entre variáveis de determinada população ou fenômeno. Sob o ponto de abordagem, a pesquisa pode ser classificada como qualitativa, pois se deseja analisar informações e opiniões de um instrumento chave, além de considerar uma relação dinâmica entre o mundo real e o sujeito. O método de pesquisa deste trabalho é um Survey exploratório, uma vez que envolve o questionamento direto das pessoas cujo comportamento que deseja conhecer e analisar por meio da atividade de elicitação de requisitos de alto nível, bem como verificar o comportamento de alguns ambientes existentes no mercado se atendem ou não esses requisitos, os ambientes avaliados foram: Kahoot, GoConqr, Busuu e o Duolingo [Martins et al. 2013].

\subsection{Ambientes avaliados}

Kahoot é uma plataforma gratuita, prática e simples de uso educacional que foi desenvolvida pelos cientistas da Universidade de Ciência e Tecnologia Norueguesa. Disponível no site http://www.kahoot.it, a ferramenta é fundamentada com base nos elementos dos jogos, com diversos tipos de modalidades para ser aplicado no processo de ensino, permite a criação de questionários em grupos, como quizzes, testes e entre outras. O sistema possibilita aos alunos um feedback e ao professor uma avaliação da aprendizagem em tempo real, usa tabela de pontuação, ranqueamento para motivar a competição entre os mesmos [Costa et al. 2017].

De acordo com os próprios desenvolvedores da ferramenta, pode ser usado para estimular a aprendizagem por meio de trabalhos em equipe, revisar assuntos que promovam uma maior interação entre os alunos, proporcionando discussões e campeonatos, e, por fim, realizar testes de forma mais motivadora.

GoConqr é uma plataforma de aprendizado que está acessível em http://www.goconqr.com/pt, sendo habilitado na elaboração de quizzes, na produção 
VIII Congresso Brasileiro de Informática na Educação (CBIE 2019)

Anais do XXV Workshop de Informática na Escola (WIE 2019)

de mapas cognitivos, além de oferecer ferramentas educacionais, dentre outros. Capaz de ser aplicado da mesma maneira que uma rede social comunicativa é utilizada. A plataforma é uma avançada técnica de conhecimento e conversação com aplicabilidades singulares. Essa tecnologia proporciona um lugar de aprendizado online que viabiliza a revisão dos conteúdos com a finalidade de preparar-se em prol dos exercícios avaliativos. Também oferece aos seus usuários, ensinamentos variados, como esquemas psicológicos, informações, flashcards, entre outros. O GoConqr autoriza que os resultados sejam aplicados em um grupo com os demais usuários. Os visitantes dessa plataforma não são obrigados a fazer login, muito menos possui algum cadastro para visualizar os resultados compartilhados pelos participantes [GoConqr 2018].

Nesse sistema, são utilizados elementos dos jogos tais como: pontos (scores) que permite ver os dados numéricos que são calculados; emblemas (badges) para engajar os participantes; sistemas de classificação que são os indicadores qualitativos; tabela de líderes que serve para medir o grau de finalizar uma etapa, dentre outros.

O Busuu proporciona uma aprendizagem entre falantes e aprendizes, com um objetivo importante na parte da escrita, pois possibilita aos seus usuários aprender a língua escolhida através da escrita, leitura e fala, com a ajuda de pessoas que são falantes fluentes do idioma [Castro 2012].

Trata-se de uma plataforma para o aprendizado de línguas, na qual os usuários podem ajudar os outros a melhorar suas habilidades. Essa ferramenta disponibiliza cursos em 12 idiomas, com diferentes níveis de acesso: iniciante, básico, intermediário e avançado, focado em cursos para viajantes e negócios.

O Duolingo [Duolingo 2018] é mais um exemplo de uma das plataformas gamificadas educacionalmente. Neste ambiente, cada etapa é uma atividade para ser realizada pelo estudante. No momento que uma termina, a próxima já é liberada e assim sucessivamente, colaborando para a aprendizagem do discente. Dessa forma, é composto com lições que são separadas por tópicos, onde os mesmos vão do nível básico ao mais complexo. Tem sido analisado como um importante método a ser usado pelos professores, pois é um relevante sistema para o uso na disciplina da língua inglesa. Das atividades disponíveis no programa, é possível encontrar vários meios que viabilizem um ensino aprendizagem atrativo e dinâmico [Silva et al. 2016]. Objetiva melhorar a aprendizagem com a utilização dos elementos de pontuação e tabelas de líderes, deixando, assim, o processo de ensino motivador.

\subsection{Procedimentos de execução}

Uma vez concluída a elicitação das ferramentas gamificadas foi necessário que critérios comparativos fossem elencados de modo estes servirem de elemento condutor para o processo de comparação. Dessa forma, tendo como base o referencial teórico, foi possível identificar alguns requisitos de alto nível que serviram de critérios para a análise comparativa entre os ambientes gamificados baseados em leaderboards.

Estes requisitos incluem: i) estimular o engajamento dos alunos na resolução dos problemas; ii) o ambiente deve ser composto de etapas que instigue o aluno no processo de aprendizagem; iii) fornecer o feedback ao aluno sobre seu desempenho a partir da tabela de pontuação (rankings diários, semanais e geral); iv) o ranking deve apresentar características motivacionais para os alunos, evitando desta forma o desinteresse devido 
VIII Congresso Brasileiro de Informática na Educação (CBIE 2019)

Anais do XXV Workshop de Informática na Escola (WIE 2019)

à exposição de informação; v) o sistema deve utilizar os leaderboards com o objetivo de esforço pelo aluno e não com capacidade e aptidões; vi) possibilitar mais chances de vitória para os alunos aumentando sua motivação; vii) ser disponibilizado de forma online e gratuito para a utilização; e viii) ser disponibilizado no idioma Português Brasileiro.

Logo em seguida, as ferramentas foram avaliadas em um grupo focal formado por oito especialistas em Computação e Educação, no qual utilizaram as aplicações, refletiram sobre a experiência e, logo em seguida responderam a Survey na seguinte escala para verificar se o ambiente atendia (A) atendia parcialmente (AP) e não atendia (N) aos requisitos.

\section{Resultados e discussão}

A dinâmica utilizada para o estudo comparativo é baseada na construção de um plano cartesiano em que cada um dos ambientes é avaliado à luz de cada um dos requisitos de alto nível citados na seção anterior, mostrando se os mesmos foram ou não atendidos. A Tabela 1 apresenta a síntese da comparação feita entre os ambientes gamificados.

Tabela 1. Resumo da análise comparativa dos ambientes gamificados baseados em leaderboards

\begin{tabular}{|l|l|l|l|l|}
\hline Requisitos & Kahoot & GoConqr & Busuu & Duolingo \\
\hline $\begin{array}{l}\text { 1. Estimular o engajamento dos alunos na } \\
\text { resolução dos problemas. }\end{array}$ & $\mathrm{A}$ & $\mathrm{A}$ & $\mathrm{A}$ & $\mathrm{A}$ \\
\hline $\begin{array}{l}\text { 2. O ambiente deve ser composto de eta- } \\
\text { pas que instigue o aluno no processo de } \\
\text { aprendizagem. }\end{array}$ & $\mathrm{A}$ & $\mathrm{A}$ & $\mathrm{A}$ & $\mathrm{A}$ \\
\hline $\begin{array}{l}\text { 3. Fornecer o feedback ao aluno so- } \\
\text { bre seu desempenho a partir da tabela de } \\
\text { pontuação (rankings diários, semanais e } \\
\text { geral). }\end{array}$ & $\mathrm{AP}$ & $\mathrm{N}$ & $\mathrm{A}$ \\
\hline $\begin{array}{l}\text { 4. O ranking deve apresentar carac- } \\
\text { terísticas motivacionais para os alunos, } \\
\text { evitando desta forma o desinteresse de- } \\
\text { vido à exposição de informação. }\end{array}$ & $\mathrm{A}$ & $\mathrm{N}$ & $\mathrm{N}$ & $\mathrm{AP}$ \\
\hline $\begin{array}{l}\text { 5. O sistema deve utilizar os leaderbo- } \\
\text { ards com o objetivo de esforço pelo aluno } \\
\text { e não com capacidade e aptidões. }\end{array}$ & $\mathrm{A}$ & $\mathrm{N}$ & $\mathrm{N}$ & $\mathrm{AP}$ \\
\hline $\begin{array}{l}\text { 6. Possibilitar mais chances de } \\
\text { vitória para os alunos aumentando } \\
\text { sua motivação. }\end{array}$ & $\mathrm{A}$ & $\mathrm{AP}$ & $\mathrm{AP}$ & $\mathrm{A}$ \\
\hline $\begin{array}{l}\text { 7. Ser disponibilizado de forma online e } \\
\text { gratuita para a utilização. }\end{array}$ & AP & AP & AP & AP \\
\hline $\begin{array}{l}\text { 8. Ser disponibilizado no idioma Por- } \\
\text { tuguês Brasileiro. }\end{array}$ & NA & A & A & A \\
\hline
\end{tabular}

Legenda: $\mathrm{A}=$ Atendido / AP = Atendido Parcialmente $/ \mathrm{NA}=$ Não Atendido

A partir da interpretação da Tabela 1, pode-se perceber que todas as ferramentas avaliadas conseguem estimular o engajamento do aluno na resolução de problemas e que 
VIII Congresso Brasileiro de Informática na Educação (CBIE 2019)

Anais do XXV Workshop de Informática na Escola (WIE 2019)

seus ambientes são compostos de etapas que instiguem o aluno no processo de aprendizagem.

Entre as ferramentas avaliadas em relação aos critérios de fornecer feedback ao aluno sobre seu desempenho a partir da tabela de pontuação (rankings diários, semanais e geral) e apresentar um ranking com características motivacionais para os alunos, evitando desta forma o desinteresse devido à exposição de informação, duas delas (GoConqr e Busuu) não atendem esses quesitos. O Kahoot atende parcialmente o critério de fornecer feedback ao aluno por apresentar um ranking (diário) após a aplicação de um quiz, mas não fornece rankings semanais e nem gerais, pois sua dinâmica são competições rápidas sugeridas em sala de aula. Mesmo com essa limitação, esse ranking motiva os alunos pela competência em si, mas principalmente pelo fato de não expor os últimos colocados no ranking, apresentando apenas o Top 5. O Duolingo, por sua vez, fornece esses rankings como forma de acompanhar o desempenho em línguas diariamente, mensalmente e geral. Porém, seus rankings apresentam características desmotivacionais por expor indivíduos nas últimas colocações.

Como as ferramentas GoConqr e Busuu não apresentam rankings, não foi possível verificar se os sistemas utilizam esses rankings com objetivo de esforço pelo aluno e não com capacidades e aptidões. Já as demais ferramentas que possuem rankings estimulam esse esforço. Mesmo as duas ferramentas citadas não apresentando rankings, ambas apresentam quizzes que possibilitam chances de vitória para alunos aumentando sua motivação.

Outro critério avaliado foi se a ferramenta é disponibilizada de forma online e gratuita para a utilização. Todas as ferramentas avaliadas possuem uma versão premium e outra gratuita. O GoConqr apresenta uma versão gratuita para seus usuários criar e compartilhar recursos públicos ilimitados e $50 \mathrm{MB}$ de armazenamento de upload de mídia. Em sua versão premium ( $\$ 2,75 /$ mês), os conteúdos/recursos são compartilhados particularmente e possui 1GB de armazenamento de mídia para upload. O Busuu também possui uma versão gratuita e outra premium ( $\mathrm{R}$ \$ 9,99/mês). Em ambas, é possível realizar exercícios interativos e quizzes divertidos, porém só na versão paga é possível aperfeiçoar suas habilidades com unidades avançadas e entre outras funções se destaca também a opção de baixar todas as lições e aprender onde estiver de forma off-line.

No Duolingo, por sua vez, na versão paga (\$6,99/mês) é possível remover anúncios, baixar lições no celular, conseguir uma recuperação gratuita de ofensiva por mês e apoiar nossa missão. O Kahoot Plus está disponível para uma taxa de desconto de $\$$ 9,95/mês com um número ilimitado de participantes que podem se inscrever durante um experimento.

Em relação ao ambiente ser disponibilizado no idioma Português Brasileiro, apenas a ferramenta Kahoot não atende esse requisito. As demais, duas delas (Duolingo e Busuu) já se esperava que atendessem, pois ambas têm como objetivo o ensino de línguas, inclusive para nativos brasileiros aprenderem outros idiomas. Já a ferramenta GoConqr também suporta nosso idioma, apresentando um ambiente acessível para que seus estudantes desenvolvam os principais conteúdos e assuntos de interesse. 
VIII Congresso Brasileiro de Informática na Educação (CBIE 2019)

Anais do XXV Workshop de Informática na Escola (WIE 2019)

\section{Considerações finais}

A partir do estudo realizado sobre as plataformas gamificadas, foi possível perceber que são plataformas com grandes potencialidades. Apesar de serem voltadas e oferecerem alguns recursos educacionais que façam com que os alunos possam aprender conteúdos de forma dinâmica, ainda deixam a desejar em alguns requisitos essenciais que são propostos para um sistema gamificado capaz de ser utilizado em processo mais abrangente de ensino-aprendizagem.

Portanto, mesmo não sendo definitivo, este estudo é de muita valia, uma vez que por meio dele foi possível perceber que, apesar de existirem algumas plataformas gamificadas que possibilitem uma aprendizagem para o desenvolvimento de atividades de qualquer disciplina, atualmente não há ferramentas que respondam a todos os requisitos levantados de maneira ideal.

De uma forma bastante singela e embrionária, esta pesquisa piloto apontou que, dentre as ferramentas avaliadas, o Kahoot demonstrou ser um pouco mais eficaz que as demais, podendo ser considerada a que possui maior potencial a ser explorado em cenários educacionais. Por fim, como forma de verificar as impressões obtidas deste estudo recomenda-se como proposta de continuidade da pesquisa a realização de um experimento para verificar a motivação dos participantes em responder questões utilizando os leaderboards, atestando ou não a sua viabilidade, implicando ou não no desenvolvimento de um sistema que atenda aos requisitos educacionais e de gamificação com potencial de ser utilizado em qualquer assunto.

\section{Referências}

Aires, S. and Leite, J. C. (2017). Técnicas de motivação para colaboração no moodle imd. In Anais do VII Workshop sobre Aspectos da Interação Humano-Computador para a Web Social, pages 43-52. SBC.

Camboim, S. P. (2017). Investigando o perfil de alunos evadidos em uma disciplina introdutória do curso de ciência da computação da uepb. trabalho de conclusão de curso universidade estadual da paraíba do curso de licenciatura em computação, patos - pb.

Castro, C. H. S. (2012). Aplicativos gratuitos de ensino de inglês para android: breve relato de testes. In Anais do Congresso Nacional Universidade, EAD e Software Livre, volume 1 .

Costa, C. H. C., Dantas Filho, F. F., and Moita, F. (2017). Marvinsketch e kahoot como ferramentas no ensino de isomeria. Holos, 1:31-43.

Duolingo (2018 (Acesso Abril 8, 2018)). Ensino personalizado. http: //www . duolingo.com/info.

Fregoneis, J. G. P. (2002). Estudo do desempenho acadêmico nos cursos de graduação dos Centros de Ciências Exatas e de Tecnologia da Universidade Estadual de Maringá: período 1995-2000. $\mathrm{PhD}$ thesis, Dissertação (Mestrado em Engenharia da Produção). Universidade Federal de Santa Catarina.

GoConqr (2018 (Acesso Abril 20, 2018)). Site do GoConqr. https://www . goconqr.com/pt-BR. 
VIII Congresso Brasileiro de Informática na Educação (CBIE 2019)

Anais do XXV Workshop de Informática na Escola (WIE 2019)

Martins, R. A., Mello, J. B. P., and Turrioni, C. H. (2013). Guia para elaboração de monografia e TCC em engenharia de produção. Editora Atlas SA.

Oliveira, J. F., Libâneo, J. C., and Toschi, M. S. (2017). Educação escolar: políticas, estrutura e organização. Cortez Editora.

Silva, J. S., Santana, J. S., and Alves, V. L. S. (2016). O uso do aplicativo duolingo: Uma forma inclusiva de ensinar e aprender línguas. In Anais do II Congresso Internacional de Educação Inclusiva. Editora Realize. 